\title{
LA TENEUR EN GAZ ET SON INFLUENCE SUR LES PROPRIÉTÉS DU BEURRE
}

\author{
par \\ J.-M. DE MAN
}

L'introduction des méthodes continues et le barattage sous pression réduite ont créé un surcroît d'intérêt pour la phase gazeuse du beurre, sa détermination, et l'influence sur ses propriétés. Le gaz peut être présent dans le beurre sous deux formes, premièrement comme gaz libre dans ses cavités de forme plus ou moins régulières, et deuxièmement comme gaz dissous, pour la plupart dans l'huile du beurre. On parle souvent de l'air dans le beurre, mais cette expression n'est pas exacte, parce qu'on a démontré que la composition du gaz de beurre était différente de celle de l'air. Cette différence peut être le résultat de réactions purement chimiques, comme celles causées par la neutralisation de la crème avec du carbonate de soude, ou de caractère biologique, causées par la croissance des microbes dans la crème ou dans le beurre. D'après les observations de ShEBanin, la teneur en oxygène du beurre diminue remarquablement pendant l'entreposage. D'après Dyer, la quantité de bioxyde de carbone dans le beurre en entreposage croît jusqu'à une valeur maximum et diminue ensuite progressivement. Quelques-unes des méthodes de fabrication continue du beurre permettent l'introduction d'une quantité mesurée de gaz et, généralement, on utilise du gaz inerte plutôt que de l'air.

\section{Méthodes de détermination}

Les méthodes employées pour la détermination du gaz dans le beurre peuvent être divisées en trois groupes :

1. Celles où le gaz est séparé du beurre et mesuré directement;

2. Celles où le poids spécifique du beurre est mesuré et la teneur en gaz calculée à partir de cette valeur ;

3. Celles où le beurre est soumis à une pression réduite et la teneur en gaz calculée d'après l'expansion observée.

Groupe 1. Монr et Eysank ont décrit une méthode où l'échantillon de beurre est chauffé dans un bain de glycérol ou d'éthylène glycol; le gaz qui s'échappe est rassemblé dans un entonnoir renversẻ portant un tube gradué. La quantité de gaz est ainsi déterminée directement. Une modification de cette méthode en utilisant de l'eau bouillie a été utilisée par De MAN et Wood (1958a). 
Une autre méthode directe a été décrite par ARUP et VAN Gilmour. La teneur en gaz était représentée par la différence du volume spécifique, avant et après fusion. Le volume spécifique du beurre originel était trouvé en pesant un volume connu de beurre dans un anneau métallique. Le volume spécifique après fusion était déterminé en chauffant du beurre dans un récipient gradué, le gaz échappé étant remplacé par l'eau d'une burette.

Dans la méthode dite "de centrifugation" décrite par Rosen et LANNHARD et employée par SANDELIN et par MenNiCke (1942), un morceau de beurre est mis dans un butyromètre ou appareil similaire, qui est ensuite rempli complètement avec de l'eau et fermé par un bouchon. Après chauffage, le butyromètre est mis dans un centrifugeur et le gaz se rassemble dans la partie graduée. On déplace le bouchon sous l'eau et on fait la lecture de la quantité de gaz pendant que l'appareil se trouve dans l'eau.

Groupe 2. Mennicke (1943) a indirectement déterminé la teneur en gaz à partir du poids spécifique du beurre. Il pesait le beurre dans un cône métallique de volume connu. Cette méthode simple a aussi été utilisée par DE MAN et Wood (1958a), qui ont démontré une haute corrélation entre le poids spécifique et la teneur en gaz du beurre. Mennicke (1943) et Schulz ont démontré que les différences de la teneur en gaz ont une influence bien plus importante sur le poids spécifique du beurre que les variations en poids spécifiques des autres éléments constituants. ScHulz a déterminé le poids spécifique en plaçant des morceaux de beurre dans des cylindres remplis de mélanges d'alcool et d'eau, de poids spécifiques variés. Le cylindre dans lequel le beurre reste flottant contient le mélange du même poids spécifique que lui. Posтнumus a mesuré le poids spécifique en plaçant un morceau de beurre sur le point aigu d'un hydromètre spécial. En mettant des poids sur l'hydromètre, suspendu dans l'eau, il fut possible de calculer le poids spécifique du beurre.

Groupe 3. RAHN et MонR ont décrit une méthode basée sur l'expansion du gaz dans le beurre, sous vide. La méthode a été améliorée par Guthrie et par Loftus Hills et Conochie. Le beurre est pressé dans un cône métallique et pesé. Le cône est ensuite garni d'un bonnet en verre et mis dans une bouteille munie d'un tube gradué. Le montage de l'appareil se fait sous l'eau bouillie afin d'exclure complètement l'air. L'appareil est ensuite chauffé dans un bain d'eau afin de fondre le beurre et de rassembler le gaz dans le bonnet. Quand on applique le vide au tube gradué, une augmentation de volume en résulte, ce qui permet de calculer la teneur en gaz de l'échantillon. D'après Loftus Hills et Conochie, 
il est possible de déterminer avec cette méthode la teneur en gaz libre et dissous.

\section{Avantages et désavantages de ces méthodes}

Les méthodes des groupes 1 et 2 ne possèdent pas une grande précision et la méthode "au vide " est probablement plus précise que toutes les autres. ScHulz a attiré notre attention sur l'inhomogénéité du beurre en ce qui concerne le gaz. Ceci a été démontré aussi par De MaN et Wood (1958a). Ils ont fait douze déterminations de la teneur en gaz dans un seul échantillon de beurre et ils ont trouvé des résultats variant de 2,4 à $3,6 \%$. A cause de cette variation dans la distribution du gaz, il ne semble pas pratique d'employer une méthode très précise et compliquée. Une meilleure manière d'obtenir de bons résultats est de faire plusieurs essais et de calculer la moyenne.

Loftus Huls et CoNochie ont indiqué que le beurre contient du gaz libre et du gaz dissous. Il n'existe que peu de renseignements sur la solubilité des gaz dans les huiles. Schaffer et Haller ont rendu compte des solubilités des gaz dans des huiles végétales et Morelu et al. ont publié des résultats sur l'huile de beurre. Loftus Hills et CoNochie présument que dans les méthodes directes, par exemple celle de MoHr et Eysank, une partie du gaz dissous est libéré pendant le chauffage de l'échantillon. Ceci, cependant, parait être extrêmement invraisemblable. Dans leur mémoire, Loftus Hinls et Conochie déclarent que la solubilité des gaz dans le gras solide est tellement faible qu'on peut la négliger, et ils présument que le beurre à $20^{\circ} \mathrm{C}$. contient environ $50 \%$ de gras solide. Dans ce cas, en chauffant le beurre jusqu'à son point de fusion, on rend disponible $50 \%$ de plus de gras liquide pour dissoudre le gaz, ce qui pourrait être plus que la quantité libérée par l'accroissement de la température. Parce qu'il n'existe pas assez de renseignements sur la solubilité des gaz dans l'huile de beurre, il nous faut présumer que les méthodes directes indiquent le gaz libre seulement.

Les méthodes des groupes 1 et 2 sont faciles à réaliser et n'exigent que de l'outillage simple. Un désavantage de la méthode de Mohr et Eysank est l'usage de grandes quantités de glycérol ou de glycol. Ce désavantage est surmonté par l'emploi d'eau bouillie. La plupart des méthodes du groupe 2 pour obtenir le poids spécifique sont recherchées parce que le seul travail nécessaire est de peser un volume connu de beurre.

\section{La teneur en gaz du beurre}

McDowall a énuméré les résultats obtenus dans divers pays du monde. La moyenne de la teneur en gaz semble être environ 
de 3 à $5 \%$. Il existe, cependant, une grande variabilité dans les valeurs citées. Des teneurs en gaz aussi élevées que 14,9\% ont été rapportées par Pickerill et GuTHRIE. Du beurre avec une teneur en gaz aussi élevée parait spongieux. DE MAN et Wood (1958a), ont rapporté une moyenne de $3,8 \%$ de gaz dans des beurres canadiens non empaquetés. Le beurre empaqueté avait une teneur en gaz fortement réduite, $2 \%$ seulement en moyenne. Ces résultats sont en contradiction avec ceux de BARNICOAT, qui rapportait une augmentation en gaz causée par l'empaquetage. D'après MCDowall le malaxage du beurre peut augmenter la teneur en gaz. La teneur en gaz du beurre d'été est plus élevée que celle du beurre d'hiver (MoDow ALL, DE MAN et WoOD, 1958 a). Les beurres continus Alfa, Gold'n Flow et Creamery Package ont une teneur en gaz extrêmement basse; le beurre Fritz par contre, a une teneur en gaz plus élevée que le beurre normal (environ 6-9\%, ScHulz).

La réduction de la teneur en gaz par barattage et malaxage sous vide est délimitée par le niveau du vide appliqué. Il est bien possible de réduire la teneur en gaz du beurre jusqu'à moins de $1 \%$.

\section{Influence de la teneur en gaz sur les propriétés du beurre}

La teneur en gaz détermine la propriété adhésive du beurre. Quand le beurre contient une faible quantité de gaz, comme le beurre Gold'n Flow, il a une apparence luisante et une texture compacte; ce beurre n'adhère pas à l'emballage de parchemin. Le beurre normal a une tendance prononcée à adhérer au parchemin. DE MAN et Wood (1958b) ont démontré que l'augmentation de la quantité de gaz dans le beurre Gold'n Flow causait une augmentation de la propriété adhésive.

La fermeté du beurre est diminuée par la présence de gaz. Ce fait a été démontré par $\mathrm{DE}$ MAN et Wood (1958b) en utilisant l'augmentation de la teneur en gaz du beurre continu, et par Swarting et al., Ystgaard et Korvald, et Pedersen en comparant des beurres normaux à ceux malaxés sous vide. SToRgards et HuMBERT n'ont pas trouvé de différence remarquable entre le beurre normal et celui malaxé sous vide. Il est bon de reconnaître les difficultés dans la détermination de ces différences, parce qu'il est bien possible que les dimensions des bulles de gaz et la durée du malaxage soient ne pas uniformes dans les diverses expériences, ce qui peut donner des résultats douteux.

D'après Pedersen, le malaxage sous vide n'a aucune influence sur la tendance à laisser exsuder l'huile. Conformément à cette opinion, De MAN et Wood (1958c) ne pouvaient démontrer aucune différence en exsudation d'huile entre des beurres contenant beaucoup ou peu de gaz. MoHr et BAUR, au contraire, ont rapporté 
qu'une haute teneur en gaz augmentait la tendance à exsudation d'huile. Swartling et al. ont remarqué qu'un niveau de vide trop bas pendant le malaxage faisait apparaître des gouttelettes d'huile sur la surface du beurre.

Il y a controverse quant à l'influence de la teneur en gaz du beurre sur sa qualité de conservation. ARUP et VAN GILMOUR ont rapporté que le beurre d'une faible teneur en gaz se conservait mieux. Humbert a remarqué moins d'oxydation dans l'intérieur du beurre malaxé sous vide, mais aucune différence n'était trouvée à l'examen organoleptique. D'après STORgARDS et AULE, le barattage sous vide n'influence pas la susceptibilité au développement des saveurs oxydées ou à la croissance des microbes. D'après Swarthing et al., le barattage sous vide n'a aucune influence sur la saveur du beurre, mais la qualité de conservation pourrait être légèrement améliorée.

YsTGAaRD et KORVALD n'ont pu démontrer aucune différence en ce qui concerne saveur et qualité de conservation entre les beurres normaux et ceux barattés sous vide, à l'exception des beurres à indice d'iode plus élevé que 32,6. D'après Pedersen, aucune différence n'est décelable entre le beurre normal et celui baratté sous vide.

\section{(Université d'Alberta, Edmonton, Canada.)}

\section{BIBLIOGRAPHIE}

P. S. Arup et G. Van Gilmour. J. Dep. Lands. Agr. (Ireland), 32, 257, 1933.

C. R. Barnicoat. New Zealand J. Sci. Technol,, 29A, 193, 1947.

J. M. DE MAN et F. W. Wood. Milchwissenschaft, 13, 75, 1958 a.

J. M. DE MAN et F. W. Wood. J. Dairy Sei., 41, 360, 1958b.

J. M. DE Man et F. W. Wood. J. Dairy Sci., 41, 369, 1958c.

D. C. Dyer. J. Agr. Res., 6, 927, 1916.

E. S. Guthrie. J. Dairy Sci., 13, 461, 1930.

E. S. Humbert. Can. Dairy Ice Cream J., 35, (8), 27, 1956.

G. Loftus Hills et J. CoNoohie. J. Council Sci. Ind. Research (Aust.), 18, 366,1945 .

F. H. MoDowall. The buttermaker's manual. Wellington, New Zealand University Press. 1953.

U. Mennicke. Milchwirtsch. Forsch, 21, 252, 1942.

U. Mennicke. Milchwirtsch. Forsch., 21, 261, 1943.

W. Монr et E. EYSANk. Fette u. Seifen, 3, 143, 1943.

W. МоHr et K. BAUR. Milchwissenschaft, 3, 17, 1948.

D. B. Morell, J. Conochie et G. Loftus Hills. J. Council Sci. Ind. Research (Aust.), 19, 190, 1946.

A. H. Pedersen. Milchwissenschaft, 11, 265, 1956.

H. M. Piokerill et E. S. Guthrie. Cornell Agr. Exp. Sta. Bull., 355, 1915.

E. G. Pont. J. Couneil Sci. Ind. Research (Aust.), 18, 53, 1945. 
G. Posthumus Neth. Milk Dairy J., 9, 107, 1955.

O. RAHN et W. MoHr. Milchwirtsch. Forsch., 1, 213, 1924.

A. Rosen et M. LANnhard. S'venska Mejeritidn., 28, 353, 1936.

A. E. SANdelin. Meijerit, Aikakaush, 1. 111, 1939.

P. S. Schaffer et H. S. Haller. Oil and Soap, 20, 161, 1943.

P. Shebanin. Mol. Prom., 13, 35, 1952.

M. E. SchuLz. Milchwissenschaft, 3, 196, 1948.

T. Storgards et O. Aule. 14th Int. Dairy Congr., 2, 448, 1956.

P. Swartling, T. Olsson et A. B. Buhrgard. 14th Int. Dairy Congr., 2, 473, 1956.

O. M. Ystgaard et T. Korvald. Rapport no. 51 de l'Institut de laiterie de l'Université norvégien d'agriculture.

\section{LE BROME DANS LE LAIT(1)}

par

\section{Giorgio CURLI et Dino COPPINI}

Comme le nombre d'auteurs qui se sont occupés de la recherche et du dosage du brome dans le lait pur est très restreint, nos connaissances sur ce sujet sont très limitées.

A. Damiens (2) a trouvé 0 mgr. 3 de brome par 100 grammes de lactose contenu dans le lait; A. Casıni (3) a dosé d'après la méthode de I. BeLlucci (4) une quantité de brome variant de 18,4 jusqu'à 24,1 $\gamma \%$. Récemment F. Guffroy (5) a exposé les données relatives à 15 échantillons examinés, et il a trouvé 6 à $25 \gamma$ de brome pour 100 centimètres cubes de lait.

Par conséquent, on trouve que le nombre des échantillons examinés est plutôt limité, et qu'il est assurément très éloigné de la limite qu'on désirerait pour le chimiste bromatologue.

Le problème offre un grand intérêt car le nombre des conservateurs contenant du brome est désormais considérable. Lorsque, dans la recherche de ces conservateurs, on suit une méthode spécifique, la présence du brome du lait ne gêne pas; cependant, si l'on exécute la recherche du brome des conservateurs sur les cendres, on a affaire à des difficultés insurmontables.

Lorsqu'il s'agit de lait condensé ou en poudre, le brome contenu dans le lait dont on est parti, se concentre et arrive même à des valeurs élevées.

Les auteurs indiqués ont reconnu dans le brome contenu dans

[1] Ann. Fals. et Fraudes, 1957, No 587.588, 397.

(2) A. Damiens, Bull. des Sc. Pharm., IV, 163, 1921.

(3) A. Casini. Ann. Chim. App., XXXVI, 219. 1946.

(4) I. Bellucci. Gazetta Chim. It., LXXII, 507. 1942.

(5) F. Guffrey. Ann. Fals. Fraudes, XI.VII, 423. 1954. 